1994;51:767-771). (Reprints: Dr Ney, Dept Neurology, EEG Laboratory, Long Island Jewish Medical Center, New Hyde Park, NY 11042).

COMMENT. The exact cause of the cerebellar atrophy was not determined. The partial seizures, the phenytoin, or both factors were involved. Other known causes of cerebellar atrophy had been excluded. Monitoring the serum phenytoin may have provided a correlation between MRI ratings of atrophy and the possible effects of a chronic level of toxicity. Long-term treatment with phenytoin is generally safe provided optimal therapeutic levels are maintained. Current emphasis on monotherapy may lead to dosage increments above acceptable levels, with the attendant risk of a chronic subtle ataxia, especially in patients with refractory epilepsies.

\title{
APLASTIC ANEMIA WITH ETHOSUXIMIDE
}

An 8-year-old girl who developed aplastic anemia after 8 months ethosuximide therapy for absence seizures is reported from the Children's Medical College of Virginia, Richmond, VA. Blood counts and liver enzymes had been monitored 3 months before admission and were normal. She presented with fatigue, headache, streptococcal pharyngitis, hematuria, bruising, and petechiae. Allogeneic bone marrow transplantation was required and the child recovered. Without further AED therapy she has only occasional "staring spells" and the EEG is normal. A total of 8 cases of ethosuximide-associated aplastic anemia have been reported, and 5 died. (Massey GV, Myer EC et al. Aplastic anemia following therapy for absence seizures with ethosuximide. Pediatr Neurol July 1994;11:59-61). (Respond: Dr Massey, PO Box 980121, MCV Station, Richmond, VA 23298).

COMMENT. Ethosuximide-related aplastic anemia is rare but has a high mortality. Monthly blood counts have been recommended, but their predictive value is questioned by the authors. Fever, rash, bruising, and petechiae should certainly require immediate investigation.

\section{HEADACHE}

\section{THE EEG IN CHRONIC HEADACHE EVALUATION}

A retrospective analysis of records of 312 children with chronic headache and review of EEGs in 257 are reported from the Tel Aviv Medical Center and University, Israel. Headache was classified as migraine in 143 (55\%); classic migraine in 12 and common migraine in 121 . The mean age at time of EEG was 9 years. The EEG was normal in $80 \%$. Epileptiform activity occurred in $12 \%$ and slowing in $8 \%$. Response was higher to hyperventilation in non-migraine patients and to photic stimulation in those with migraine. The incidence of epileptic EEGs was $11 \%$ in both migraine and tension type headaches; it was $26 \%$ and significantly higher in 15 children with chronic headache described as "very brief," occurring predominantly in girls, several times a week, and without family history for migraine. Prevalence of epilepsy in families of patients with epileptic EEGs did not differ from the total group. Of six children with epileptiform EEGs who were treated with AEDs, 4 responded and had no further headaches and 2 were not benefited. Of 17 children with focal EEG abnormalities, 9 had head CTs, 1 had an arachnoid cyst, and 3 had sinusitis. The authors conclude that the EEG may be of value in some children 